\title{
Improvement of Scalp Condition and Quality of Life through Proper Skin Care of Dry Scalp
}

\author{
Mika Oshima1, Maiko Sogawa1, Hiroshi Matsunaka², Yumi Murakami², Yumiko Saya², \\ Takashi Sugita ${ }^{3}$, Yoshihiro Matsudate', Nozomi Fukui1, Kazutoshi Murao1, Yoshiaki Kubo1* \\ ${ }^{1}$ Department of Dermatology, Institute of Health Biosciences, The University of Tokushima Graduate School, \\ Tokushima, Japan \\ ${ }^{2}$ Tokiwa Pharmaceutical Co., Ltd., Tokyo, Japan \\ ${ }^{3}$ Department of Microbiology, Meiji Pharmaceutical University, Tokyo, Japan \\ Email: ${ }^{*}$ kubo@tokushima-u.ac.jp
}

Received 11 July 2014; revised 3 August 2014; accepted 6 September 2014

Copyright (C) 2014 by authors and Scientific Research Publishing Inc.

This work is licensed under the Creative Commons Attribution International License (CC BY). http://creativecommons.org/licenses/by/4.0/

(c) (i) Open Access

\section{Abstract}

Introduction: Appropriate skin care for dry scalp includes treatment for dandruff and itchiness. However, little is known about the appropriate washing methods for subjects with dry scalp. Methods: A scalp moisturizing lotion was used for 8 weeks on 30 outpatients at the Tokushima University Hospital who had dry scalp. All subjects were given the same hair shampoo/conditioner and instructions on how to wash their hair. Scalp symptoms were observed before and after the testing period. Indigenous bacteria were collected from the scalp for quantitative assessment. A survey was conducted to assess the quality of life (QOL). Results: All scalp condition items (dryness, scales and desquamation, itchiness, excoriation, and erythema) improved significantly after the testing period; the levels of Malassezia and bacterial colonization also decreased significantly. The QOL evaluation indicated significant improvements in "symptoms", "emotions", and "global" measures. A correlation was found between the extent of dryness at the start of the test and the level of Malassezia colonization, as well as QOL scores. Conclusion: Using a scalp moisturizing lotion, washing hair with a shampoo and conditioner that cause low irritation levels, and using a hair washing method that avoids irritation led to improvement in scalp condition, QOL, and patient satisfaction.

\section{Keywords}

Atopic Dermatitis, Seborrheic Dermatitis, Moisturizer, Malassezia, Bacteria, qPCR

\footnotetext{
${ }^{*}$ Corresponding author.
}

How to cite this paper: Oshima, M., et al. (2014) Improvement of Scalp Condition and Quality of Life through Proper Skin Care of Dry Scalp. Journal of Cosmetics, Dermatological Sciences and Applications, 4, 284-292. 


\section{Introduction}

The scalp is a region where a relatively large number of hair glands exist and the amount of sebum excretion is large [1], making it difficult to dry out. Dry symptoms of the scalp are often triggered, however, due to underlying ailments such as atopic dermatitis, seborrheic dermatitis, or psoriasis vulgaris, as well as low humidity during winter, cooling and heating in highly sealed living environments, or wrong skin care from using an excessive amount of detergent. On the other hand, a rise in sebum excretion induces increased number of indigenous bacteria and changes in bacterial flora [2], resulting in an increased amount of inflammation and desquamation due to dermatitis seborrheica or turnover disorder, which can lead to dryness of the scalp. Therefore, skin problems can readily occur in the scalp, but the awareness about the importance of skin care in this region is low when compared with the face.

In this study, a moisturizing lotion for the scalp was used on test subjects with ailments such as atopic dermatitis, seborrheic dermatitis, and psoriasis vulgaris; the symptoms of these subjects had stabilized because of continuous treatment, but they had dry scalp. Furthermore, their hair shampoo and hair conditioner were replaced with those that had no antibacterial agents or cooling ingredients and cause low skin irritation. The hair washing method used in the present study, which involved brushing through the hair with the fingertips, facilitated improvements with regards to excessive washing or insufficient rinsing. After the testing period, skin findings, the levels of Malassezia and bacteria, and patient quality of life (QOL) were assessed, correlations between these parameters were verified, and the clinical efficacy was examined.

\section{Methods}

\subsection{Subjects}

The test was conducted on 30 outpatients of Tokushima University Hospital with ailments such as atopic dermatitis, seborrheic dermatitis, and psoriasis vulgaris; the symptoms of these subjects had stabilized because of continuous treatment, but they had dry scalp. They did not have any severe symptoms of inflammation, papular eruptions, or any serious underlying ailments and were determined to be unsuitable for this study by their physicians-in-charge. They were also provided information about the study before the test was performed, and written informed consent was obtained from those who voluntarily desired to participate in the study.

The following patients were considered eligible for participation: patients with ailments such as atopic dermatitis, seborrheic dermatitis, and psoriasis vulgaris whose symptoms related to their underlying disease had stabilized due to treatment but who had dry scalp; outpatients who were able to wash their hair once a day as a general rule (in case of those whose customary frequency of washing hair was once every 2 days prior to participation in this study, the frequency was set to once every 2 days); and subjects 20 years of age or older, irrespective of gender.

The exclusion criteria were as follows: skin symptoms such as contact dermatitis from formulation ingredients of the product under study, or similar constituents; severe inflammation or rash on the scalp, which would have made the use of the product under study inappropriate; underlying disease symptoms that had not been stabilized; inability to attend follow-up examination 8 weeks later; hair washing frequency of less than once every 2 days; pregnancy or lactation; and other reasons for which patients were deemed unsuitable for participation in this study by their physician-in-charge.

\subsection{Implementation Period and Implementation Facility}

Eight consecutive weeks were set for each patient from April 2013 to October 2013 for testing. The testing facility was the Department of Dermatology at the Tokushima University Hospital, and the test was implemented by a resident dermatologist of the Department of Dermatology. Furthermore, study approval was received from the Ethics Committee of the hospital prior to the implementation of the test (Approval Number: 1656).

\subsection{Test Samples}

The product under study is a moisturizing lotion for the scalp developed by Tokiwa Pharmaceutical Co., Ltd., intended to improve dryness symptoms of the scalp. The product contains moisturizing components such as hyaluronic acid and antioxidative components such as tocophenol. Furthermore, the moisturizing lotion contained no fragrances or synthetic coloring and was prepared to ensure that the induction of primary irritation of 
the skin or allergic reaction would be low. Furthermore, the hair shampoo and conditioner, which were used in combination, were also devised to ensure that skin irritation would be low, with no disinfectant agents or cooling ingredients included in the product. The scalp moisturizing lotion (NOV ${ }^{\circledR}$ Scalp Lotion, Noevir Co., Ltd., Hyogo, Japan) is a product made by partially improving a formula for the purpose of increasing moisturizing effects of a product that is already available on the market in Japan. The hair shampoo (NOV ${ }^{\circledR}$ Hair Shampoo D, NOV ${ }^{\circledR}$ Hair Shampoo DS, Noevir Co., Ltd.) and hair conditioner (NOV ${ }^{\circledR}$ Hair Conditioner, Noevir Co., Ltd.) are all currently available on the market. In terms of the Pharmaceutical Affairs Law in Japan, the scalp moisturizing lotion is classified as a quasi-drug, while the hair shampoo and conditioner are categorized as cosmetic products. In the 48-hour closed patch tests, there was no difference in the degree of stimulation when compared to the simultaneously tested petrolatum (Kenei Pharmaceutical Co. Ltd., Osaka, Japan) and purified water (Kenei Pharmaceutical Co. Ltd., Osaka, Japan) and therefore, the shampoo and conditioner were confirmed to be highly safe. The hair shampoo is available as a refreshing type $\left(\mathrm{NOV}^{\circledR}\right.$ Hair Shampoo D) and moisturizing type (NOV ${ }^{\circledR}$ Hair Shampoo DS), and were selected by the subjects according to the length of their hair and preference. The hair conditioner was used according to the preference of the subjects and was used, for instance, by women with longer hair.

\subsection{Usage}

The treatment (orally taken or externally applied) of the patients' underlying ailments prior to the commencement of the test, as well as treatment for any complication, was continued throughout the study, with no change in the type, usage, or dosage. However, if changes in the previously established treatment were necessary, and the physician-in-charge considered that such changes would affect the test, the patient was treated as an exception. The subjects washed their scalp and hair using the shampoo, as well as hair conditioner if preferred, once a day when they bathed, according to the scalp skin care method instructed by the physician-in-charge. After washing their hair, the subjects applied approximately $2 \mathrm{~mL}$ of moisturizing lotion over the entire scalp, while the amount of hair shampoo and hair conditioner used for shoulder-length hair was approximately $5 \mathrm{~mL}$. Hair washing was to be performed once a day; however, if the subjects customarily washed their hair once every 2 days, then they were instructed to continue washing their hair at that frequency throughout the study. Any other skin care products being used prior to the study, such as hairdressing products or coloring agents, were allowed if they had been determined not to irritate the scalp.

\subsection{Instructions on Hair Washing Method}

The physician-in-charge provided each subject instructions on how to wash their hair, according to the contents in Table 1.

\subsection{Observation and Evaluation Methods}

The physician-in-charge entered the identification number, age, sex, complications, combined medications for use during the test period (medication name, usage, and dosage) of each anonymous subject (linkable anonymity) on the case card of each subject and observed the skin symptoms of the scalp.

1) Skin observations

a) The extent of dryness, itchiness, scratch marks, and erythema were judged according to the following criteria and rated in scores as follows:

\section{Table 1. Washing method.}

1) Shampoo

Place some shampoo on the palm of the hand and spread it on both hands softly, and then apply it to the back of the head and foam it towards the side of the head. Shampoo the hair from the back of the head and the top of the head to the front of the head gently, using the balls of the fingers, not the nails.

2) Rinsing
Warm water does not reach the scalp only by pouring it from the top, which leaves shampoo ingredients on the scalp.
Raise the roots using the finger comb to allow warm water to reach the roots.


4. Severe: Significant symptoms visible.

3. Intermediate: Symptoms are clearly visible.

2. Slight: Symptoms are somewhat visible.

1. Minute: Very little symptoms are visible.

0 . None: No symptoms are visible.

b) The desquamation and scales observed to be falling off the scalp when the hair on the top of the head is parted, was judged according to the following criteria and rated in scores as follows:

3. Advanced: Considerable amount confirmed.

2. Intermediate: Easily confirmed.

1. Slight: Little amount confirmed.

0 . None: None confirmed or very little confirmed with none attaching to the hair.

2) Collection of fungus from the scalp and quantitative analysis of Malassezia and bacterial DNA

a) Collection

The swab portion of an aseptic cotton swab with a test tube (JCB Industry Ltd., Tokyo, Japan) was immersed in distilled water (Otsuka Pharmaceutical Co., Ltd., Tokyo, Japan) and the swab portion was rubbed against the scalp within a specific area $(3 \mathrm{~cm} \times 3 \mathrm{~cm})$ by making 15 roundtrips in the $\mathrm{X}$-axis direction and 15 roundtrips in the Y-axis direction, while rotating the swab to collect fungus from the scalp. The swab was then stored in a refrigerator at $4^{\circ} \mathrm{C}$.

b) Quantitative analysis of Malassezia and bacteria by qPCR

Fungal and bacterial DNA was extracted directly from the swab portion. The amounts of Malassezia and bacterial DNA were quantified using real-time PCR (Applied Biosystems, CA, USA) with a TaqMan probe [3] [4].

3) Safety

Details of adverse effects (symptoms, extent, whether or not the subject should stop participating in the test, treatments, and outcomes) were entered on the case card. When a causal relationship with the product under the study was confirmed, such reactions were considered adverse reactions.

4) QOL evaluation

The Skindex-16, a measure of specific skin ailments, was used to investigate the QOL of test subjects at the start and end of the test [5]. Furthermore, Skindex-16 was used to investigate the scalp condition.

5) Survey

The extent of "dryness" and "itchiness" of the scalp as felt by the subjects were investigated with a set of multiple choice questions at the start and end of the test.

a) Dryness

- Dry;

- Slightly dry;

- Not dry;

- Do not know.

b) Itchiness

- Itchy to the point it is not possible to refrain from scratching;

- Notice scratching unconsciously;

- Scratch slightly here and there;

- Scratch slightly but can refrain from scratching;

- Feel no itch.

\subsection{Statistical Analysis}

Correlations between the scalp condition scores at the start of the test, scores for the respective observation items, the levels of colonization by Malassezia and bacteria, Skindex-16 measures, and correlations between quantitative changes in the scores for the respective observation items before and after the test were examined using Spearman's rank-order correlation coefficient. Furthermore, whether any significance existed between the scores of respective observation items for scalp condition before and after the test, the levels of colonization by Malassezia and bacteria, and changes in the Skindex-16 measures, were analyzed using a paired t-test or Wilcoxon signed-rank test. A chi-square analysis of the survey response items was performed. A p-value $<0.05$ was considered statistically significant. 


\section{Results}

\subsection{Cases and Test Subject Background}

A total of 30 subjects (average age: $47 \pm 14$ years) consisting of 15 men (average age: $49 \pm 15$ years) and 15 women (average age: $46 \pm 14$ years) were included in the present study. Diagnostic descriptions included atopic dermatitis, seborrheic dermatitis, psoriasis vulgaris, and eczema capitis, among others.The following complications were observed: hypertension in 2 patients, and prostatic hypertrophy, pollinosis, asthma, Kimura's disease, depression, hyperthyroidism, follicular mucinosis, and reflux esophagitis in one patient each. Concomitant drugs included both topical agents and oral agents. The topical agents consisted of steroids, immunosuppressants, and others, which were used by 22, 2, and 12 patients, respectively. The oral agents consisted of anti-allergic agents, steroids, immunosuppressants, and others, which were used by 18, 5, 3, and 16 patients, respectively. During the trial period, there were no changes in the agents used (Table 2).

\subsection{Safety}

Out of the 30 cases, one was excluded because the subject with seborrheic dermatitis did not come in for an examination at the end of the test, and another case was considered to have deviated because the subject with psoriasis vulgaris suspended the use of Antebate lotion, which resulted in the occurrence of itchiness. The use of the product under study was suspended 8 days after the start of the test in one subject with seborrheic dermatitis, as papular eruption and itchiness occurred. No treatment was provided for this condition, but the symptom disappeared naturally 1 week later so the use of the product was restarted, with no problem occurring during the

Table 2. Characteristics of subjects.

\begin{tabular}{|c|c|c|c|}
\hline & & Men & Women \\
\hline \multirow{8}{*}{ Age, years } & $21-30$ & 1 & 2 \\
\hline & $31-40$ & 4 & 5 \\
\hline & $41-50$ & 3 & 4 \\
\hline & $51-60$ & 3 & 1 \\
\hline & $\geq 61$ & 4 & 3 \\
\hline & Total, $\mathrm{n}$ & 15 & 15 \\
\hline & Mean \pm SD & $49 \pm 15$ & $46 \pm 14$ \\
\hline & Mean \pm SD & \multicolumn{2}{|c|}{$47 \pm 14$} \\
\hline Diagnostic name & Atopic dermatitis & \multicolumn{2}{|c|}{9} \\
\hline (some overlapping) & Seborrheic dermatitis & \multicolumn{2}{|c|}{3} \\
\hline & Psoriasis (psoriasis vulgaris, pustular psoriasis) & \multicolumn{2}{|c|}{5} \\
\hline & Eczema & \multicolumn{2}{|c|}{5} \\
\hline & Prurigo & \multicolumn{2}{|c|}{4} \\
\hline & Alopecia areata & \multicolumn{2}{|c|}{4} \\
\hline & Contact dermatitis & \multicolumn{2}{|c|}{1} \\
\hline & Scleroderma & \multicolumn{2}{|c|}{1} \\
\hline Complication & Hypertension & \multicolumn{2}{|c|}{2} \\
\hline (some overlapping) & $\begin{array}{l}\text { Prostatic hypertrophy, pollinosis, asthma, Kimura’s disease, depression, } \\
\text { hyperthyroidism, follicular mucinosis, reflux esophagitis }\end{array}$ & \multicolumn{2}{|c|}{1 each } \\
\hline Concomitant drug & Steroid & \multicolumn{2}{|c|}{22} \\
\hline External & Immunosuppressant & \multicolumn{2}{|c|}{2} \\
\hline & Other than the above & \multicolumn{2}{|c|}{12} \\
\hline Concomitant drug & Anti-allergic agent & \multicolumn{2}{|c|}{18} \\
\hline Internal & Steroid & \multicolumn{2}{|c|}{5} \\
\hline & Immunosuppressant & \multicolumn{2}{|c|}{3} \\
\hline & Other than the above & \multicolumn{2}{|c|}{16} \\
\hline
\end{tabular}


remaining 6 weeks. This instance was determined to have had "no" causal relationship with the product under study.

Itchiness in the head region occurred in 2 subjects with atopic dermatitis at 15 days and 27 days after starting the use of the product; the use of the product under study was suspended in these subjects. In one of these subjects, the symptom did not improve after the use of the product was suspended; however, this subject also presented a rash in regions other than the head. For this reason, the symptom was determined to have had no relationship with the product under study. No symptom improvement occurred in the other case, and while seasonal deterioration of the skin symptom was considered possible, since the causal relationship could not be ruled out, the determination was a "yes" for the existence of adverse effects. Twenty-six cases except for the 4 abovementioned cases were aggregated.

\subsection{Skin Observations}

The scores at the start and end of the test for the respective observation items of scalp symptoms were: Dryness (1.3 \pm 0.2 and $0.4 \pm 0.1)$; scale and desquamation $(0.9 \pm 0.2$ and $0.5 \pm 0.2)$; itchiness (2.2 \pm 0.2 and $1.1 \pm 0.2)$; scratches (1.4 \pm 0.2 and $0.7 \pm 0.2)$; and erythema ( $2.0 \pm 0.1$ and $1.2 \pm 0.2)$, with all item scores showing a significant decline (Figure 1).

\subsection{Colonization by Malassezia and Bacteria}

The levels of colonization by Malassezia and bacteria decreased significantly from the start to the end of the 8week testing period (Figure 2).

\subsection{QOL}

Of the measures of Skindex-16, which are "Symptoms", "Emotions", "Functioning”, and "Global", the item scores of "Symptoms", "Emotions", and "Global" significantly decreased from the start to the end of the 8-week testing period (Figure 3).

\subsection{Survey}

The evaluations of dryness at the start and the end of the test were "dry" (5/1), "slightly dry" (8/3), and "not dry" (7/13); changes in the shape of dry-skin patches were considered to indicate improvement. The evaluations of itchiness at the start and the end of the test were "Itchy to the point it is not possible to refrain from scratching" (5/1), "Notice scratching unconsciously" (10/4), "Scratch slightly here and there" (5/10), "Scratch slightly but can refrain from scratching" (5/5), and "Feel no itch" (1/6), with significant changes (Table 3).

\subsection{Statistical Analysis (Correlation)}

The correlations between the scalp condition scores at the start of the test, scores for the respective observation items, the levels of colonization by Malassezia and bacteria, and the Skindex-16 measures are shown in Table 4. The correlations of quantitative changes in the scores for the respective observation items relating to the condition of the scalp before and after the test are shown in Table 5.

"Dryness" at the start of the test was found to be positively correlated with the observation items of the scalp and Skindex-16 measures "symptoms" and "global", while the level of Malassezia colonization was found to be negatively correlated. "Scales and desquamation" was found to be negatively correlated with the levels of colonization by Malassezia and bacteria; however, the correlation was only statistically significant between "scales and desquamation" and the level of Malassezia colonization.

Furthermore, with respect to the change in scores of respective observation items relating to the condition of the scalp ( $\Delta$ at the end—at the beginning), " $\Delta$ dryness" was positively correlated with all observation items.

\section{Discussion}

In 26 cases, the scalp symptoms before the test showed significant improvements by the end of the testing period, indicating that scalp skin care as performed in this study (moisturizing, use of hair shampoo and hair conditioners that present low levels of irritation, as well as the hair washing method that presents low levels of irritation) 


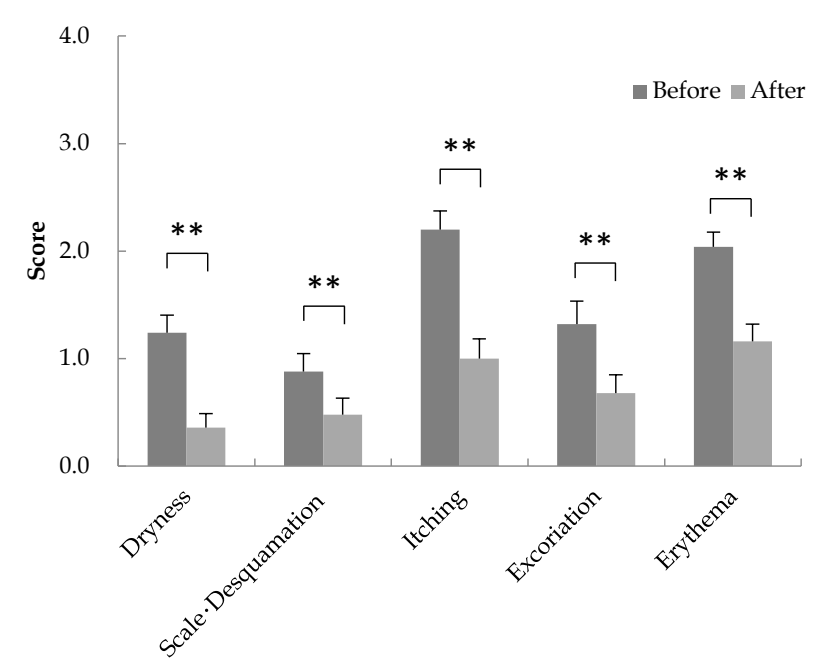

Figure 1. Skin findings.
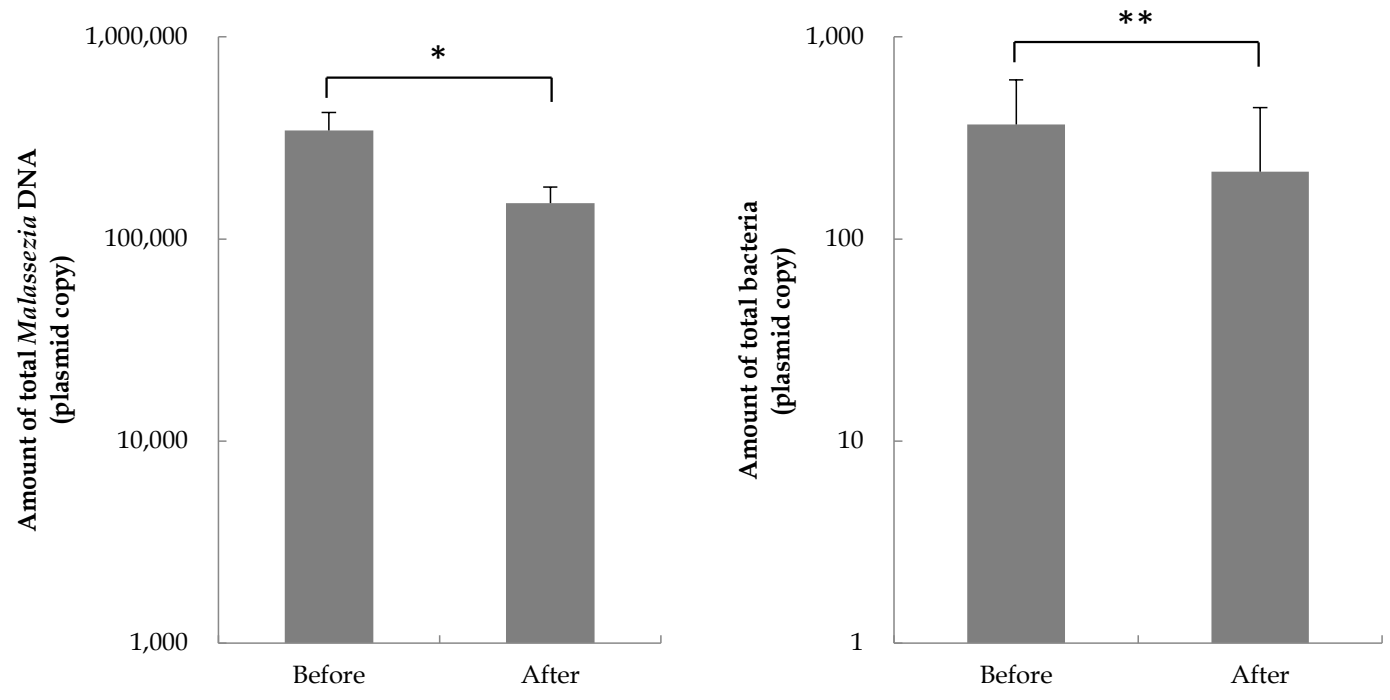

Figure 2. Evaluation of the levels of colonization by Malassezia and bacteria.

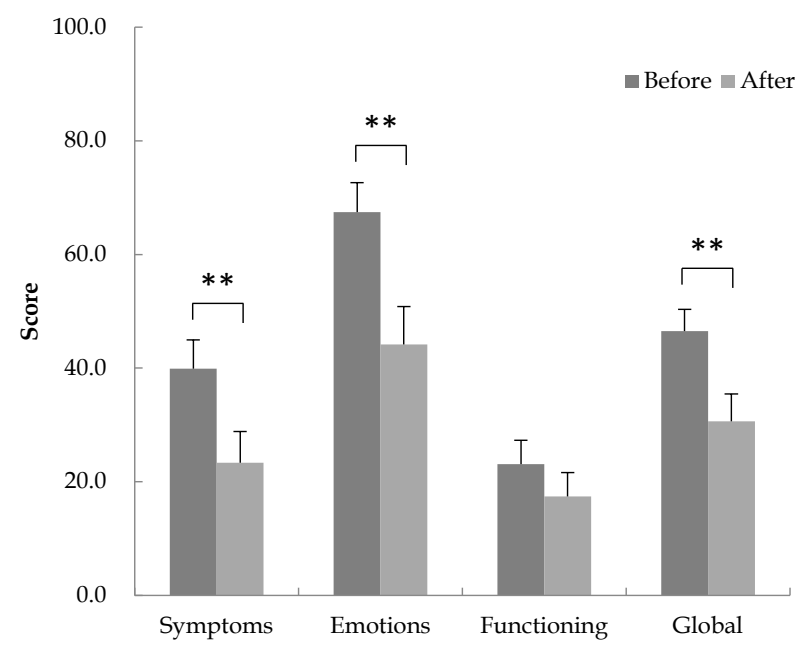

Figure 3. Evaluation of QOL score. 
Table 3. Evaluation of severity of dryness and itchiness.

\begin{tabular}{|c|c|c|c|c|c|c|c|c|c|c|c|}
\hline \multirow{2}{*}{$\begin{array}{c}\text { Dryness } \\
\mathrm{n}=25 \\
\text { Before }\end{array}$} & \multicolumn{2}{|c|}{ Dry } & \multicolumn{2}{|c|}{ Slightly dry } & \multicolumn{2}{|c|}{ Not dry } & \multicolumn{2}{|c|}{ Do not know } & & & \multirow{2}{*}{$\begin{array}{c}\begin{array}{c}\mathrm{p} \text {-value } \\
\chi^{2} \text {-test }\end{array} \\
0.059\end{array}$} \\
\hline & 5 & $20 \%$ & 8 & $32 \%$ & 7 & $28 \%$ & 5 & $20 \%$ & & & \\
\hline After & 1 & $4 \%$ & 3 & $12 \%$ & 13 & $52 \%$ & 8 & $32 \%$ & & & \\
\hline $\begin{array}{c}\text { Itchiness } \\
n=26\end{array}$ & \multicolumn{2}{|c|}{$\begin{array}{l}\text { Itchy to the point it is } \\
\text { not possible to refrain } \\
\text { from scratching }\end{array}$} & \multicolumn{2}{|c|}{$\begin{array}{l}\text { Notice scratching } \\
\text { unconsciously }\end{array}$} & \multicolumn{2}{|c|}{$\begin{array}{l}\text { Scratch slightly here } \\
\text { and there }\end{array}$} & \multicolumn{2}{|c|}{$\begin{array}{l}\text { Scratch slightly but } \\
\text { can refrain from } \\
\text { scratching }\end{array}$} & \multicolumn{2}{|c|}{ Feel no itch } & $\begin{array}{c}\mathrm{p} \text {-value } \\
\chi^{2} \text {-test }\end{array}$ \\
\hline Before & 5 & $19 \%$ & 10 & $38 \%$ & 5 & $19 \%$ & 5 & $19 \%$ & 1 & $4 \%$ & 0.033 \\
\hline After & 1 & $4 \%$ & 4 & $15 \%$ & 10 & $38 \%$ & 5 & $19 \%$ & 6 & $23 \%$ & \\
\hline
\end{tabular}

Table 4. Correlation between skin findings, amount of colonization of the total Malassezia and total bacteria, and quality of life score.

\begin{tabular}{|c|c|c|c|c|c|c|c|c|c|c|c|}
\hline & & \multicolumn{4}{|c|}{ Skin findings } & \multicolumn{2}{|c|}{ Level of colonization } & \multicolumn{4}{|c|}{ Quality of life score } \\
\hline & & $\begin{array}{c}\text { Scale- } \\
\text { Desquamation }\end{array}$ & Itching & Excoriation & Erythema & $\begin{array}{c}\text { Total } \\
\text { Malassezia }\end{array}$ & $\begin{array}{c}\text { Total } \\
\text { Bacteria }\end{array}$ & Symptoms & Emotions & Functionin & S Global \\
\hline \multirow{3}{*}{ Dryness } & $\mathrm{r}$ & 0.55 & 0.48 & 0.48 & 0.42 & -0.44 & 0.03 & 0.57 & 0.20 & 0.19 & 0.40 \\
\hline & $\mathrm{p}$ & 0.002 & 0.008 & 0.007 & 0.020 & 0.015 & 0.859 & 0.001 & 0.307 & 0.336 & 0.030 \\
\hline & $\mathrm{n}$ & 30 & 30 & 30 & 30 & 30 & 30 & 29 & 29 & 29 & 29 \\
\hline \multirow{3}{*}{$\begin{array}{c}\text { Scale- } \\
\text { Desquamation }\end{array}$} & $\mathrm{r}$ & - & 0.02 & 0.16 & 0.18 & -0.58 & -0.15 & 0.34 & 0.34 & 0.26 & 0.38 \\
\hline & $\mathrm{p}$ & - & 0.901 & 0.405 & 0.335 & 0.001 & 0.423 & 0.069 & 0.071 & 0.173 & 0.044 \\
\hline & $\mathrm{n}$ & - & 30 & 30 & 30 & 30 & 30 & 29 & 29 & 29 & 29 \\
\hline \multirow{3}{*}{ Itching } & $\mathrm{r}$ & - & - & 0.83 & 0.45 & 0.15 & 0.19 & 0.36 & 0.09 & 0.12 & 0.24 \\
\hline & $\mathrm{p}$ & - & - & 0.000 & 0.013 & 0.417 & 0.305 & 0.055 & 0.647 & 0.528 & 0.203 \\
\hline & $\mathrm{n}$ & - & - & 30 & 30 & 30 & 30 & 29 & 29 & 29 & 29 \\
\hline \multirow{3}{*}{ Excoriation } & $\mathrm{r}$ & - & - & - & 0.47 & 0.17 & 0.40 & 0.29 & 0.06 & 0.18 & 0.24 \\
\hline & $\mathrm{p}$ & - & - & - & 0.009 & 0.358 & 0.027 & 0.120 & 0.749 & 0.349 & 0.203 \\
\hline & $\mathrm{n}$ & - & - & - & 30 & 30 & 30 & 29 & 29 & 29 & 29 \\
\hline \multirow{3}{*}{ Erythema } & $\mathrm{r}$ & - & - & - & - & -0.18 & 0.05 & 0.31 & -0.02 & -0.07 & 0.03 \\
\hline & $\mathrm{p}$ & - & - & - & - & 0.341 & 0.803 & 0.100 & 0.904 & 0.706 & 0.861 \\
\hline & $\mathrm{n}$ & - & - & - & - & 30 & 30 & 29 & 29 & 29 & 29 \\
\hline
\end{tabular}

Table 5. Correlations between skin findings.

\begin{tabular}{|c|c|c|c|c|c|}
\hline & & \multicolumn{4}{|c|}{ Skin findings } \\
\hline & & $\Delta$ Scale-Desquamation & $\Delta$ Itching & $\Delta$ Excoriation & $\Delta$ Erythema \\
\hline \multirow{3}{*}{$\Delta$ Dryness } & $\mathrm{r}$ & 0.40 & 0.50 & 0.44 & 0.40 \\
\hline & $\mathrm{p}$ & 0.045 & 0.009 & 0.026 & 0.045 \\
\hline & $\mathrm{n}$ & 26 & 26 & 26 & 26 \\
\hline \multirow{3}{*}{$\Delta$ Scale-Desquamation } & $\mathrm{r}$ & - & 0.09 & 0.02 & 0.24 \\
\hline & $\mathrm{p}$ & - & 0.662 & 0.915 & 0.239 \\
\hline & $\mathrm{n}$ & - & 26 & 26 & 26 \\
\hline \multirow{3}{*}{$\Delta$ Itching } & $\mathrm{r}$ & - & - & 0.48 & 0.28 \\
\hline & $\mathrm{p}$ & - & - & 0.013 & 0.164 \\
\hline & $\mathrm{n}$ & - & - & 26 & 26 \\
\hline \multirow{3}{*}{$\Delta$ Excoriation } & $\mathrm{r}$ & - & - & - & 0.21 \\
\hline & $\mathrm{p}$ & - & - & - & 0.303 \\
\hline & $\mathrm{n}$ & - & - & - & 26 \\
\hline
\end{tabular}


is effective.

The "dryness" score of the scalp at the start of the testing period had a positive correlation with all other symptomatic scores, indicating that the level of dryness was associated with a variety of other symptoms; it can be surmised that dry symptom improvement is important for scalp care. This is also supported by the fact that the " $\Delta$ dryness" had a positive correlation with the amount of change in all observation item scores from the start to the end of the testing period.

Seborrheic dermatitis has a negative effect on patients' QOL [6]. The fact that the "dryness" score was negatively correlated with the level of Malassezia colonization indicates that Malassezia prefers areas where the skin is moist and has a large quantity of sebum, and that it finds it difficult to multiply on a dry scalp. Although there was some concern that moisturizing a dry scalp may lead to bacterial multiplication, the quantities of Malassezia and bacteria decreased significantly at the end of the testing period. Moisturizing the dry scalp and washing the hair with a suitable shampoo resulted in a decrease in colonization by Malassezia and bacteria, which use sebum as their source of nutrition and produce decomposition products such as free fatty acids, possibly contributing to the improvement of symptoms such as erythema.

In conclusion, using a moisturizing lotion for dry scalp, washing hair with a shampoo and conditioner that cause low levels of irritation, and providing instructions on a hair washing method that avoids irritation led to the improvement of the scalp condition and to improved QOL and patient satisfaction. Adherence to the treatment of underlying ailments is therefore expected to improve by providing appropriate skin care to patients who have dry scalp symptoms.

\section{Declaration of Interest}

All expenses, as well as documents required, were provided for by Tokiwa Pharmaceutical Co., Ltd.

\section{References}

[1] Ebling, F.J. (1965) The Sebaceous Glands. Journal of the Society of Cosmetic Chemists, 16, 405-411.

[2] Tajima, M., Sugita, T., Nishikawa, A. and Tsuboi, R. (2008) Molecular Analysis of Malassezia microflora in Seborrheic Dermatitis Patients: Comparison with Other Diseases and Healthy Subjects. Journal of Investigative Dermatology, 128, 345-351.

[3] Sugita, T., Tajima, M., Tsubuku, H., Tsuboi, R. and Nishikawa, A. (2006) Quantitative Analysis of Cutaneous Malassezia in Atopic Dermatitis Patients Using Real-Time PCR. Microbiology and Immunology, 50, 549-552. http://dx.doi.org/10.1111/j.1348-0421.2006.tb03825.X

[4] Gao, Z., Perez-Perez, G.I., Chen, Y. and Blaser, M.J. (2010) Quantitation of Major Human Cutaneous Bacterial and Fungal Populations. Journal of Clinical Microbiology, 48, 3575-3581. http://dx.doi.org/10.1128/JCM.00597-10

[5] Higaki, Y., Kawamoto, K., Kamo, T., Horikawa, N., Kawashima, M. and Chren, M.M. (2002) The Japanese Version of Skindex-16: A Brief Quality-of-Life Measure for Patients with Skin Diseases. The Journal of Dermatology, 29, 693698. http://dx.doi.org/10.1111/j.1346-8138.2002.tb00205.x

[6] Szepietowski, J.C., Reich, A., Wesolowska-Szepietowska, E., Baran, E. and National Quality of Life in Dermatology Group (2009) Quality of Life in Patients Suffering from Seborrheic Dermatitis: Influence of Age, Gender and Education Level. Mycoses, 52, 357-363. http://dx.doi.org/10.1111/j.1439-0507.2008.01624.x 
Scientific Research Publishing (SCIRP) is one of the largest Open Access journal publishers. It is currently publishing more than 200 open access, online, peer-reviewed journals covering a wide range of academic disciplines. SCIRP serves the worldwide academic communities and contributes to the progress and application of science with its publication.

Other selected journals from SCIRP are listed as below. Submit your manuscript to us via either submit@scirp.org or Online Submission Portal.
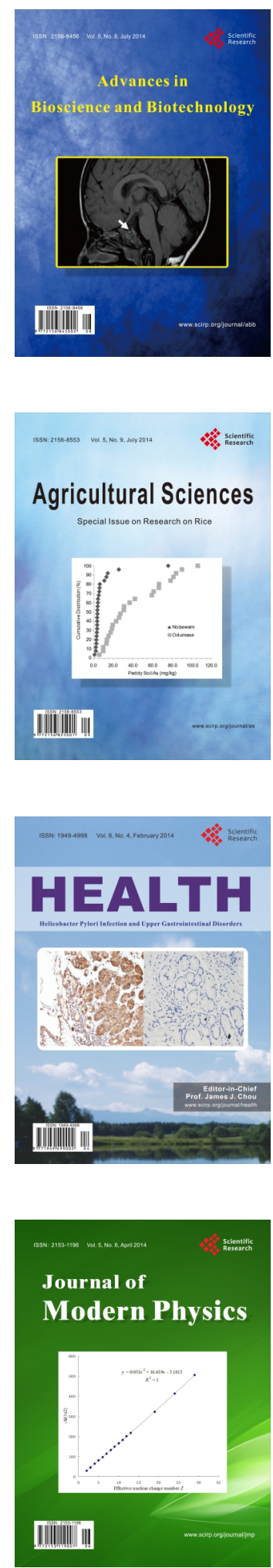
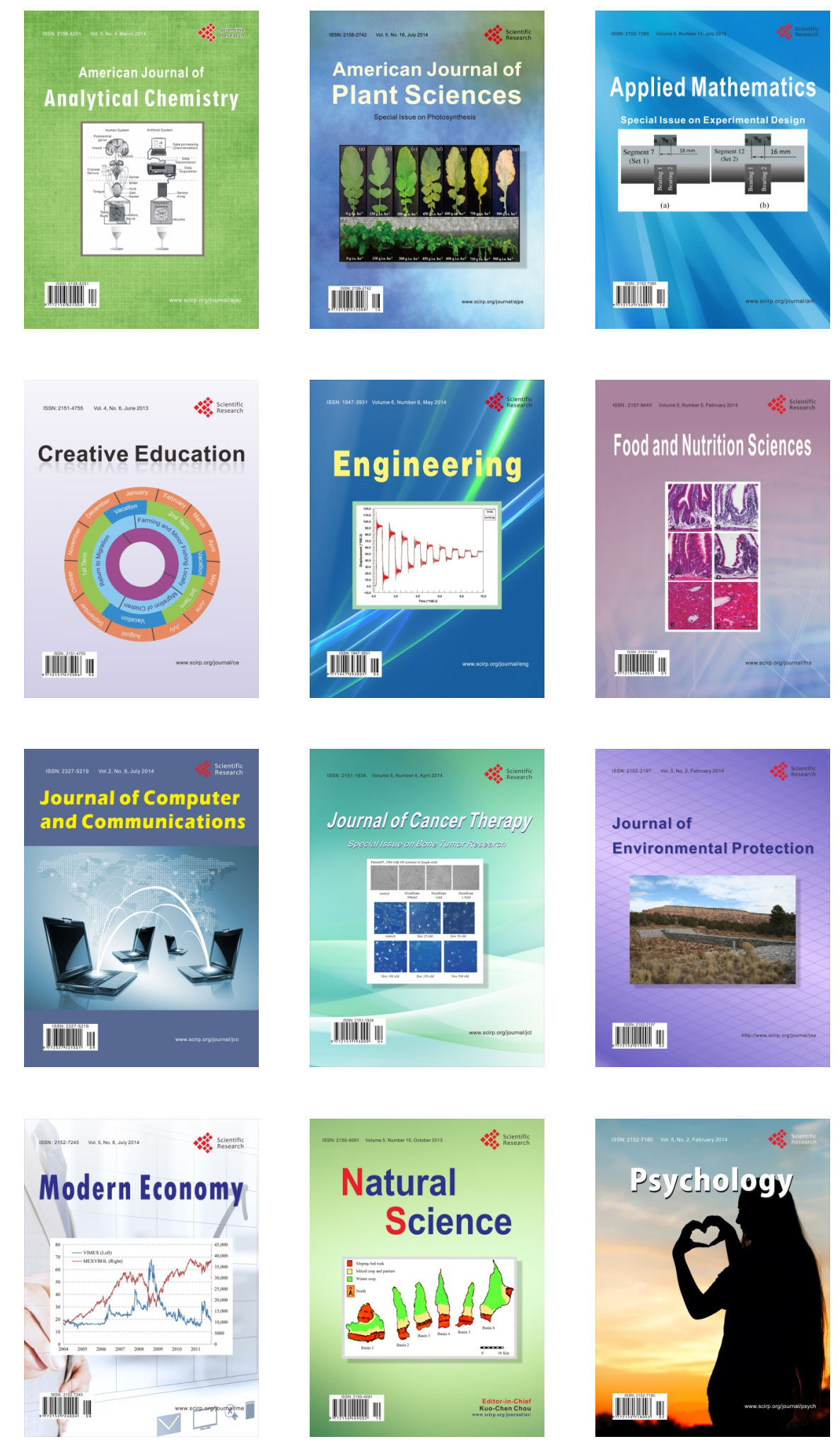